\title{
A DOMAIN CONTAINING ALL ZEROS OF THE PARTIAL THETA FUNCTION
}

\author{
VLADIMIR PETROV KOSTOV
}

\begin{abstract}
We consider the partial theta function, i.e. the sum of the bivariate series $\theta(q, z):=\sum_{j=0}^{\infty} q^{j(j+1) / 2} z^{j}$ for $q \in(0,1), z \in \mathbb{C}$. We show that for any value of the parameter $q \in(0,1)$ all zeros of the function $\theta(q,$.$) belong to$ the domain $\{\operatorname{Re} z<0,|\operatorname{Im} z| \leq 132\} \cup\{\operatorname{Re} z \geq 0,|z| \leq 18\}$.

Key words: partial theta function, Jacobi theta function, Jacobi triple product
\end{abstract}

AMS classification: 26A06

\section{INTRODUCTION}

We consider the bivariate series $\theta(q, z):=\sum_{j=0}^{\infty} q^{j(j+1) / 2} z^{j}$ for $q \in(0,1), z \in \mathbb{C}$. We regard $q$ as a parameter and $z$ as a variable. This series is convergent and defines an entire function called partial theta function. The terminology is explained by the resemblance of this formula with the one for the function

$$
\Theta^{*}(q, z):=\sum_{j=-\infty}^{\infty} q^{j(j+1) / 2} z^{j},
$$

because the latter is connected with the Jacobi theta function

$$
\Theta(q, z):=\sum_{j=-\infty}^{\infty} q^{j^{2}} z^{j}
$$

by the formula $\Theta^{*}(q, z)=\Theta\left(q^{1 / 2}, q^{1 / 2} z\right)$. The word "partial" reminds that in the formula for $\theta$ the summation is performed from 0 to $\infty$, not from $-\infty$ to $\infty$.

Studying the function $\theta$ is motivated by its applications in several domains the most recent of which concerns section-hyperbolic polynomials, i.e. real univariate polynomials of degree $\geq 2$ with all roots real and such that when their highestdegree monomial is deleted this gives again a polynomial having only real roots. The relationship between $\theta$ and such polynomials is explained in 10. Previous research on section-hyperbolic polynomials was performed in [6] and [11] which in turn was based on classical results of Hardy, Petrovitch and Hutchinson (see 4, [12. and [5]). Other domains in which the partial theta function is used are statistical physics and combinatorics (see [13]), asymptotic analysis (see [2]), Ramanujan-type $q$-series (see [14]) and the theory of (mock) modular forms (see [3]); see also [1].

In the present paper we prove the following theorem:

Theorem 1. (1) For $\operatorname{Re} z \geq 0$ the function $\theta(q,$.$) has no zeros outside the closed$ half-disk $\{\operatorname{Re} z \geq 0,|z| \leq 18\}$, for any $q \in(0,1)$. 
(2) For $\operatorname{Re} z<0$ and for any $q \in(0,1)$ the function $\theta(q,$.$) has no zeros outside$ the half-strip $\{\operatorname{Re} z<0,|\operatorname{Im} z| \leq 132\}$.

In order to explain the importance of this theorem we recall in Section 2 certain facts about the zeros of $\theta$. Then we give an example of a value of $q \in(0,1)$ for which $\theta(q,$.$) has a complex conjugate pair of zeros in the right half-plane. The$ proof of the theorem is given in Section 3 .

\section{Properties of the Function $\theta$}

In the present section we recall some results concerning the function $\theta$. We denote by $\Gamma$ the spectrum of $\theta$, i.e. the set of values of $q$ for which $\theta(q,$.$) has$ a multiple zero (the notion has been introduced by B. Z. Shapiro in [10]). The following results are proved in [7]:

Theorem 2. (1) The spectrum $\Gamma$ consists of countably-many values of $q$ denoted by $0<\tilde{q}_{1}<\tilde{q}_{2}<\cdots<\tilde{q}_{N}<\cdots<1$ with $\lim _{j \rightarrow \infty} \tilde{q}_{j}=1^{-}$.

(2) For $\tilde{q}_{N} \in \Gamma$ the function $\theta\left(\tilde{q}_{N},.\right)$ has exactly one multiple real zero $y_{N}$ which is negative, of multiplicity 2 and is the rightmost of its real zeros.

(3) For $q \in\left(\tilde{q}_{N}, \tilde{q}_{N+1}\right]$ (we set $\tilde{q}_{0}:=0$ ) the function $\theta$ has exactly $N$ complex conjugate pairs of zeros (counted with multiplicity). All its other zeros are real negative.

Remarks 1. (1) It is proved in [10] that $\tilde{q}_{1}=0.3092 \ldots$ Up to 6 decimals the first 12 spectral numbers equal (see [10])

$$
\begin{array}{llllll}
0.309249, & 0.516959, & 0.630628, & 0.701265, & 0.749269, & 0.783984, \\
0.810251, & 0.830816, & 0.847353, & 0.860942, & 0.872305, & 0.881949 .
\end{array}
$$

(2) It is shown in [7] that for $q \in\left(0, \tilde{q}_{1}\right)$ all zeros of $\theta$ are real, negative and distinct. For all $q \in(0,1)$ it is true that as $q$ increases, the values of the local minima of $\theta$ between two negative zeros increase and the values of its maxima between two negative zeros decrease. It is always the rightmost two negative zeros with a minimum of $\theta$ between them that coalesce to form a double zero of $\theta$ for $q=\tilde{q}_{N}$ and then a complex conjugate pair for $q=\tilde{q}_{N}^{+}$. For any $q \in(0,1)$ the function $\theta(q,$.$) has infinitely-many negative zeros and no positive ones; \theta(q,$.$) is$ increasing for $x>0$ and tends to $\infty$ as $x \rightarrow \infty$; there is no finite accumulation point for the zeros of $\theta(q,$.$) .$

(3) In 8 the following asymptotic expansions of $\tilde{q}_{N}$ and $y_{N}$ are given:

$$
\begin{aligned}
& \tilde{q}_{N}=1-(\pi / 2 N)+(\log N) / 8 N^{2}+O\left(1 / N^{2}\right), \\
& y_{N}=-e^{\pi} e^{-(\log N) / 4 N+O(1 / N)} .
\end{aligned}
$$

The importance of Theorem 1 lies in the fact that while the real zeros of $\theta$ remain all negative for any $q \in(0,1)$, no information was known about its complex conjugate pairs. It would be interesting to know whether all complex conjugate pairs remain (for all $q \in\left(\tilde{q}_{1}, 1\right)$ ) within some compact domain in $\mathbb{C}$ (independent of $q$ ).

Lemma 1. The function $\theta(0.73,$.$) has exactly one complex conjugate pair of zeros$ inside the open half-disk $\tilde{D}:=\{|z|<3, \operatorname{Re} z>0\}$. 
Proof. Consider the truncation of $\theta(0.73,$.$) of degree 20$ w.r.t. $x$, i.e. the polynomial $\theta_{20}:=\sum_{j=0}^{20} 0.73^{j(j+1) / 2} x^{j}$. One checks numerically (say, using MAPLE) that $\theta_{20}$ has zeros $0.03356612894 \ldots \pm 2.885381139 \ldots i$. These are the only zeros of $\theta_{20}$ in the closure of $\tilde{D}$. Numerical check shows that the modulus of the restriction of $\theta_{20}$ to the border of $\tilde{D}$ is everywhere larger than 0.016 . On the other hand the sum $\sum_{j=21}^{\infty}\left|0.73^{j(j+1) / 2} x^{j}\right|$ is $\leq \sum_{j=21}^{\infty} 0.73^{j(j+1) / 2} 3^{j}<3 \times 10^{-22}$. By the Rouché theorem the functions $\theta(0.73,$.$) and \theta_{20}$ have one and the same number of zeros inside the half-disk $\tilde{D}$.

\section{Proof of Theorem 1}

As for $q \in\left(0, \tilde{q}_{1}\right]$ all zeros of $\theta(q,$.$) are negative (see Remarks 1), we prove$ Theorem 1 only for $q \in\left(\tilde{q}_{1}, 1\right)$.

3.1. The Jacobi theta function. In the proof of Theorem 1 we use the Jacobi theta function $\Theta(q, z):=\sum_{j=-\infty}^{\infty} q^{j^{2}} z^{j}$. By the Jacobi triple product one has

$$
\Theta\left(q, z^{2}\right)=\prod_{m=1}^{\infty}\left(1-q^{2 m}\right)\left(1+z^{2} q^{2 m-1}\right)\left(1+z^{-2} q^{2 m-1}\right)
$$

from which for the function $\Theta^{*}(q, z):=\Theta\left(q^{1 / 2}, q^{1 / 2} z\right)=\sum_{j=-\infty}^{\infty} q^{j(j+1) / 2} z^{j}$ one deduces the formula

$$
\Theta^{*}(q, z)=\prod_{m=1}^{\infty}\left(1-q^{m}\right)\left(1+z q^{m}\right)\left(1+q^{m-1} / z\right) .
$$

Notation 1. We set

$$
\begin{array}{llll}
s_{m}:=1+q^{m-1} / z & t_{m}:=1+z q^{m} & , & Q:=\prod_{m=1}^{\infty}\left(1-q^{m}\right), \\
P:=\prod_{m=1}^{\infty} t_{m} & \text { and } & R & :=\prod_{m=1}^{\infty} s_{m}
\end{array} .
$$

Thus $\Theta^{*}=Q P R$.

3.2. Proof of part (1). We begin with the observation that for any factor $s_{m}$ (see (3.2) and Notation 11) one has

$$
s_{m}=1+\bar{z} q^{m-1} /|z|^{2} \text { hence }\left|s_{m}\right| \geq \operatorname{Re} s_{m} \geq 1 \text { for } \operatorname{Re} z \geq 0 .
$$

Clearly, for any factor $t_{m}$ it is true that $\left|t_{m}\right| \geq \operatorname{Re} t_{m} \geq 1$ and $\left|t_{m}\right| \geq\left|z q^{m}\right|$ for $\operatorname{Re} z \geq 0$.

Further in the proof of Theorem 1 we subdivide the interval $(0,1)$ to which $q$ belongs into intervals of the form

$$
\begin{array}{ll}
q \in(1-1 /(n-1), 1-1 / n], \quad n \in \mathbb{N}, n \geq 3 \text { and } \\
q \in\left(\tilde{q}_{1}, 1 / 2\right]
\end{array}
$$

Notation 2. We set $\theta:=\Theta^{*}-G$, where $G:=\sum_{j=-\infty}^{-1} q^{j(j+1) / 2} z^{j}$, and $u:=$ $2 e^{\left(\pi^{2} / 6\right)}=10.36133664 \ldots$ 
Remark 1. Clearly, for $|z|>1$ one has $|G| \leq \sum_{j=1}^{\infty} 1 /|z|^{j}=1 /(|z|-1)$. In particular, for $|z| \geq 18$ (resp. for $|z| \geq u$ ) one has $|G| \leq 1 / 17$ (resp. $|G| \leq 1 /(u-1)$ ).

Suppose first that $q \in(1 / 2,1)$. We show that for $|z| \geq u$, Re $z \geq 0$ one has $\left|\Theta^{*}\right|>|G|$ from which part (1) of the theorem follows.

Lemma 2. For $q \leq 1-1 / n, n \in \mathbb{N}, n \geq 2$, one has $Q \geq e^{\left(\pi^{2} / 6\right)(1-n)}$.

The lemma is a particular case of Lemma 4 in 9 .

Consider the product $P_{0}:=\prod_{m=1}^{n} t_{m}$. It follows from $\left|t_{m}\right| \geq\left|z q^{m}\right|$ that $\left|P_{0}\right| \geq$ $|z|^{n} q^{n(n+1) / 2}$. The first line of conditions (3.3) implies

$$
\begin{aligned}
\left|P_{0}\right| & \geq|z|^{n}(1-1 /(n-1))^{n(n+1) / 2}=|z|^{n}(1-1 /(n-1))^{(n-1)(n+2) / 2+1} \\
& \geq|z|^{n} 4^{-(n+2) / 2}(1-1 /(n-1)) \geq|z|^{n} 4^{-(n+3) / 2}=|z|^{n} 2^{-(n+3)}
\end{aligned}
$$

we use the inequalities

$$
(1-1 /(n-1))^{n-1} \geq 1 / 4
$$

and $1-1 /(n-1) \geq 1 / 2$ which hold true for $n \geq 3$.

Set $P_{1}:=\prod_{m=n+1}^{\infty} t_{m}$. Hence we have $\left|P_{1}\right| \geq 1,|R| \geq 1$ and

$$
\begin{aligned}
\left|\Theta^{*}\right| & =Q\left|P_{0}\right|\left|P_{1}\right||R| \geq e^{\left(\pi^{2} / 6\right)(1-n)}|z|^{n} 2^{-(n+3)} \\
& =\left(e^{\left(\pi^{2} / 6\right)} / 2^{3}\right)\left(|z| / 2 e^{\left(\pi^{2} / 6\right)}\right)^{n}
\end{aligned}
$$

Obviously, for $|z| \geq u$ one has $|z| / 2 e^{\left(\pi^{2} / 6\right)} \geq 1$. As $e^{\left(\pi^{2} / 6\right)} / 2^{3}=0.64 \ldots>1(u-1)$, one obtains the inequalities $\left|\Theta^{*}\right|>1 /(u-1) \geq|G|$ which proves part (1) of the theorem for $q \in(1 / 2,1)$ (because $u<18)$.

Suppose that $q \in\left(\tilde{q}_{1}, 1 / 2\right]$. In this case for $|z| \geq 18$ and $\operatorname{Re} z \geq 0$ one has $\left|t_{1}\right| \geq 18 \tilde{q}_{1},\left|t_{m}\right| \geq 1,\left|s_{m}\right| \geq 1$ for $m \in \mathbb{N}$ and (by Lemma 2 with $n=2$ ) $Q \geq e^{-\pi^{2} / 6}$, so $\left|\Theta^{*}\right| \geq e^{-\pi^{2} / 6} 18 \tilde{q}_{1}>1>1 /(|z|-1) \geq|G|$.

3.3. Proof of part (2). The proof of part (2) is also based on formula (3.2). We aim to show that for $\operatorname{Re} z<0$ and $|\operatorname{Im} z| \geq 132$ one has $\left|\Theta^{*}\right|>|G|$. The following technical result is necessary for the estimations and for the understanding of Figure 1

Lemma 3. For $x \in[0,0.683]$ one has $\ln (1-x) \geq-x-x^{2}$ with equality only for $x=0$.

Proof. We set $\zeta(x):=\ln (1-x)+x+x^{2}$, so $\zeta(0)=0$. As $\zeta^{\prime}=-1 /(1-x)+1+2 x=$ $x(1-2 x) /(1-x)$ which is nonnegative on $[0,1 / 2]$ and positive on $(0,1 / 2)$, one has $\zeta(x)>0$ for $x \in(0,1 / 2]$. On $(1 / 2,1)$ one has $\zeta^{\prime}<0$, so $\zeta$ is decreasing. As $\lim _{x \rightarrow 1^{-}} \zeta=-\infty, \zeta$ has a single zero on $[1 / 2,1)$. Numerical computation shows that this zero is $>0.683$ which proves the lemma.

To estimate the factor $R$ we use the following lemma:

Lemma 4. For $q \in(1-1 /(n-1), 1-1 / n], n \geq 2$, and $|\operatorname{Im} z| \geq b \geq 1.5$ one has $|R| \geq e^{-n(b+1) / b^{2}}$. 
Proof. Indeed, the condition $b \geq 1.5$ implies $1 /|z|<0.683$, so one can apply Lemma 3.

$$
\begin{aligned}
& \ln |R|=\quad \sum_{m=1}^{\infty} \ln \left|s_{m}\right| \quad \geq \sum_{m=1}^{\infty} \ln \left(1-q^{m-1} /|z|\right) \\
& \geq-\sum_{m=1}^{\infty}\left(q^{m-1} /|z|+q^{2 m-2} /|z|^{2}\right)=-1 /(1-q)|z|-1 /\left(1-q^{2}\right)|z|^{2} \\
& >\quad-(|z|+1) /(1-q)|z|^{2}
\end{aligned}
$$

which for $q \leq 1-1 / n$ is $\geq-n(|z|+1) /|z|^{2} \geq-n(b+1) / b^{2}$.

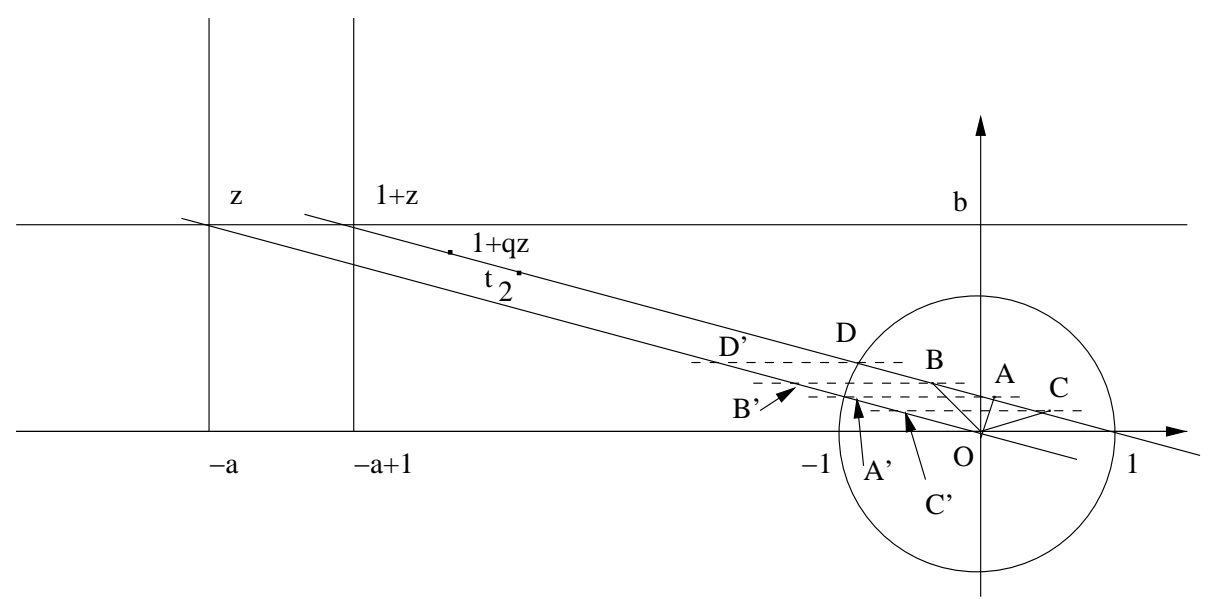

Figure 1 . The points $1+q^{m} z$.

We identify the complex numbers and the points in $\mathbb{R}^{2}$ representing them. On Fig. 1 we represent the points

$$
\begin{array}{ll}
z=-a+b i(a>0) \quad, \quad t_{0}:=1+z=1-a+b i \\
t_{1}:=1+q z=1-q a+q b i \quad \text { and } \quad t_{2}:=1+q^{2} z=1-q^{2} a+q^{2} b i .
\end{array} .
$$

The last three of them are situated on the straight line $\mathcal{L}$ passing through $1+z$ and $(1,0)$.

In what follows we assume that $b \geq 0$. The set of zeros of $\theta$ being symmetric w.r.t. the real axis this leads to no loss of generality.

The point $A \in \mathcal{L}$ is such that the segment $O A$ is orthogonal to $\mathcal{L}$. An easy computation shows that $A=\left(b^{2} /\left(a^{2}+b^{2}\right), a b /\left(a^{2}+b^{2}\right)\right)$ (one has to use the fact that the vector $(-a, b)$ is collinear with $\mathcal{L})$. The points $B$ and $C$ belong to $\mathcal{L}$. The unit circumference intersects the line $\mathcal{L}$ at $(1,0)$ and $D$. Denote by $\tilde{\Delta}$ the length $\|[A,(1,0)]\|$ of the segment $[A,(1,0)]$. The points $B$ and $C$ are defined such that

$$
\|[A, B]\|=\|[A, C]\|=0.317 \tilde{\Delta} \text { and }\|[C,(1,0)]\|=\|[D, B]\|=0.683 \tilde{\Delta} .
$$

The line $\mathcal{L}^{\prime}$ is parallel to $\mathcal{L}$. It passes through the points $z$ and $O:=(0,0)$. The points $B^{\prime}, A^{\prime}, C^{\prime}$ and $D^{\prime}$ belong to $\mathcal{L}^{\prime}$. The lines $B B^{\prime}, A A^{\prime}, C C^{\prime}$ and $D D^{\prime}$ are 
parallel to the $x$-axis. Hence the segments $[D, B],[C,(1,0)],\left[D^{\prime}, B^{\prime}\right]$ and $\left[C^{\prime}, O\right]$ are of length $0.683 \tilde{\Delta}$ while $[B, A],[A, C],\left[B^{\prime}, A^{\prime}\right]$ and $\left[A^{\prime}, C^{\prime}\right]$ are of length $0.317 \tilde{\Delta}$.

Our aim is to estimate the product $|P|:=\prod_{m=1}^{\infty}\left|t_{m}\right|=\prod_{m=1}^{\infty}\left|1+q^{m} z\right|$.

Notation 3. (1) We set

$$
|P|:=\tilde{P} P^{\ddagger} P^{\sharp} P^{\dagger},
$$

where $\tilde{P}, P^{\ddagger}, P^{\sharp}$ and $P^{\dagger}$ are the products of the moduli $\left|t_{m}\right|$ for which the point $t_{m}$ belongs to the segment $[1+z, D],[D, B],[B, C]$ and $[C,(1,0)]$ respectively.

(2) We denote by $t_{m_{0}}, t_{m_{0}+1}, \ldots$ the points $t_{m}$ belonging to the segment $[C,(1,0)]$ and we set $c_{m_{0}}:=C, c_{m_{0}+k}:=1+(C-1) q^{k}$. Hence $\left|t_{m_{0}+k}\right| \geq\left|c_{m_{0}+k}\right|$ with equality only if $t_{m_{0}}=C$.

Lemma 5. For $q \in(1-1 /(n-1), 1-1 / n], n \geq 2$, one has $P^{\dagger} \geq e^{-1.149489 n}$ and $P^{\ddagger} \geq e^{-1.149489 n}$ (where $1.149489=0.683+0.683^{2}$ ).

Proof. We notice first that the segment $[C,(1,0)]$ is of length $<0.683$. Hence $\left|t_{m_{0}+k}\right| \geq\left|c_{m_{0}+k}\right| \geq\left(1-0.683 q^{k}\right), k=0,1, \ldots$, so we can use Lemma 3 to get

$$
\begin{aligned}
\ln P^{\dagger} & \geq \ln \left(\left|c_{m_{0}}\right|\left|c_{m_{0}+1}\right| \cdots\right) \quad \geq \ln \left(\prod_{m=1}^{\infty}\left(1-0.683 q^{m-1}\right)\right) \\
& \geq-\sum_{m=1}^{\infty}\left(0.683 q^{m-1}+0.683^{2} q^{2 m-2}\right) \\
& =-0.683 /(1-q)-0.683^{2} /\left(1-q^{2}\right)>-1.149489 /(1-q) .
\end{aligned}
$$

Thus $P^{\dagger} \geq e^{-1.149489 n}$. Next, the distance between any two consecutive points $1+q^{m} z$ and $1+q^{m+1} z$ belonging to $[B, D]$ is greater than the distance between any two such points belonging to $[C,(1,0)]$. Denote by $U_{1}, U_{2}, \ldots, U_{r}$ the points $t_{m}$ belonging to the segment $[B, D]$, where $U_{1}$ (resp. $U_{r}$ ) is closest to $B$ (resp. to $D$ ). Then $\left|U_{1}\right| \geq\left|c_{m_{0}}\right|,\left|U_{2}\right| \geq\left|c_{m_{0}+1}\right|, \ldots,\left|U_{r}\right| \geq\left|c_{m_{0}+r-1}\right|$. As $\left|c_{m}\right| \leq\left|t_{m}\right|<1$ for $m \geq$ $m_{0}$, one has $P^{\ddagger} \geq\left|c_{m_{0}}\right|\left|c_{m_{0}+1}\right| \cdots\left|c_{m_{0}+r-1}\right|>\left|c_{m_{0}}\right|\left|c_{m_{0}+1}\right| \cdots \geq e^{-1.149489 n}$.

Lemma 6. There are $\leq \mu_{1}$ factors $\left|t_{m}\right|$ in $P^{\sharp}$, where

$$
\begin{aligned}
& \mu_{1}:=\ln \lambda_{1} / \ln (1 / q)+1 \quad \text { and } \\
& \lambda_{1}:=(0.634+0.683) / 0.683=1.928 \ldots
\end{aligned}
$$

with $\ln \lambda_{1}=0.6566 \ldots$ Hence

$$
P^{\sharp} \geq\left(b^{2} /\left(a^{2}+b^{2}\right)\right)^{\mu_{1} / 2}=\left(1 /\left(\beta^{2}+1\right)\right)^{\mu_{1} / 2}, \beta:=a / b .
$$

Proof. Consider the points $C^{\prime}, A^{\prime}$ and $B^{\prime}$ and the numbers $z q^{m_{1}} \in\left[B^{\prime}, A^{\prime}\right]$ and $z q^{m_{2}} \in\left[A^{\prime}, C^{\prime}\right]$ closest to $B^{\prime}$ and $C^{\prime}$ respectively. The lengths of the segments $\left[B^{\prime}, A^{\prime}\right],\left[A^{\prime}, C^{\prime}\right]$ and $\left[C^{\prime}, O\right]$ (see (3.6)) imply $\left|z q^{m_{1}}\right| /\left|z q^{m_{2}}\right| \leq \lambda_{1}$, i.e. $m_{2}-m_{1} \leq$ $\lambda_{1} / \ln (1 / q)$. The number of factors $\left|t_{m}\right|$ in $P^{\sharp}$ equals $m_{2}-m_{1}+1$ from which one deduces the first claim of the lemma. All factors $\left|t_{m}\right|$ in $P^{\sharp}$ are $<1$ and $\geq\|[O, A]\|=b /\left(a^{2}+b^{2}\right)^{1 / 2}$ from which the second claim of the lemma follows.

Remark 2. When $q \in(1 / 2,1)$ and (3.3) holds true, then $n /(n-1) \leq 1 / q<$ $(n-1) /(n-2)$. As for $x \in(0,1)$ one has $x-x^{2} / 2<\ln (1+x)<x$ (by the Leibniz criterium for alternating series), one obtains the inequalities 


$$
\begin{aligned}
& (2 n-3) / 2(n-1)^{2}=1 /(n-1)-1 / 2(n-1)^{2}<\ln (1 / q)<1 /(n-2) \text { hence } \\
& \left(\ln \lambda_{1}\right)(n-2)+1<\mu_{1} \leq \mu_{1}^{0}:=\left(\ln \lambda_{1}\right)\left(2(n-1)^{2} /(2 n-3)\right)+1 .
\end{aligned}
$$

Lemma 7. For $q \in\left(\tilde{q}_{1}, 1\right)$ and $b \geq \max (a, 132)$ one has $\left|\Theta^{*}\right|>|G|$.

Proof. Prove first the lemma for $q \in(1 / 2,1)$, see the first line of (3.3). Set again $P_{0}:=\prod_{m=1}^{n} t_{m}$. Hence

$$
\left|t_{m}\right| \geq \operatorname{Im} t_{m} \text { and }\left|P_{0}\right| \geq \prod_{m=1}^{n} b q^{m}=b^{n} q^{n(n+1) / 2} \geq b^{n} 2^{-(n+3)},
$$

see (3.4) and (3.5). With $Q, P^{\dagger}, P^{\ddagger}$ and $P^{\sharp}$ defined in Notations 11 and 3 one has

$$
\left|\Theta^{*}\right| \geq Q\left|P_{0}\right| P^{\ddagger} P^{\sharp} P^{\dagger} R \text {. }
$$

Indeed, if $b \geq 132$ and if $q$ satisfies the first line of conditions (3.3), then

$$
q^{m} \geq q^{n} \geq(1-1 /(n-1))^{n-1}(1-1 /(n-1)) \geq(1 / 4)(1-1 /(n-1)) \geq 1 / 8
$$

and $\left|b q^{m}\right| \geq\left|b q^{n}\right| \geq 132 / 8>1$. This means that all factors $\left|t_{m}\right|$ in $\left|P_{0}\right|$ are $>1$. Moreover, some factors $\left|t_{m}\right|$ with $\left|t_{m}\right|>1$ which are present in $\left|\Theta^{*}\right|$ (i.e. in $\tilde{P}$, see (3.7)) might be missing in the right-hand side of (3.9). Recall that each of the factors $P^{\dagger}$ and $P^{\ddagger}$ is minorized by $e^{-1.149489 n}$ and that $|R| \geq e^{-(b+1) n / b^{2}}$, see Lemmas 5 and 4 . Recall also that by Lemma 6 .

$$
P^{\sharp} \geq\left(1 /\left(\beta^{2}+1\right)\right)^{\mu_{1} / 2} \geq 2^{-\mu_{1} / 2} \text { (because } \beta=a / b \leq 1 \text { ) }
$$

and that $\mu_{1} \leq \mu_{1}^{0}$, see (3.8). Hence the right-hand side of (3.9) is

$$
\begin{aligned}
\geq H:= & e^{\left(\pi^{2} / 6\right)(1-n)} b^{n} 2^{-(n+3)} e^{2(-1.149489 n)} \\
& \times e^{-(\ln 2)\left(\left(\ln \lambda_{1}\right) 2(n-1)^{2} /(2 n-3)+1\right) / 2} e^{-(b+1) n / b^{2}} .
\end{aligned}
$$

Taking into account that

$$
2(n-1)^{2} /(2 n-3)=n-1 / 2+1 / 2(2 n-3),
$$

we represent the expression $H$ in the form $e^{K_{1} n+K_{0}}$, where

$$
\begin{aligned}
& K_{1}:=-\pi^{2} / 6+\ln b-\ln 2-2.298978-(\ln 2)\left(\ln \lambda_{1}\right) / 2-(b+1) / b^{2}, \\
& K_{0}:=\pi^{2} / 6-3 \ln 2+(\ln 2)\left(\ln \lambda_{1}\right) / 4-(\ln 2) / 2-(\ln 2)\left(\ln \lambda_{1}\right) / 4(2 n-3) .
\end{aligned}
$$

Recall that $n \geq 3$, see the first line of (3.3). The sum $K_{0}$ is minimal for $n=3$. For $b \geq 132$ one has $K_{1}>0$ and $\left.K_{0}\right|_{n=3}>0>-\ln (b-1)$ which implies the inequalities

$$
\left|\Theta^{*}\right| \geq Q\left|P_{0}\right| P^{\ddagger} P^{\sharp} P^{\dagger} R>1 /(b-1) \geq 1 /(|z|-1) \geq|G| .
$$

Prove the lemma for $q \in\left(\tilde{q}_{1}, 1 / 2\right]$. One has $Q \geq e^{-\pi^{2} / 6}$ (Lemma 2 with $n=$ 2), $R \geq e^{-2(b+1) / b^{2}} \geq e^{-2 \times 133 / 132^{2}}$ (Lemma 4 with $n=2$ ) and $P^{\dagger} \geq e^{-2.298978}$ (Lemma 5 with $n=2$ ). 
Lemma 8. For $q \in\left(\tilde{q}_{1}, 1 / 2\right]$ the product $P^{\ddagger} P^{\sharp}$ contains at most two factors.

Proof. Indeed, consider the line $\mathcal{L}^{\prime}$, see Fig. 1. One has $\left\|\left[O, C^{\prime}\right]\right\|=0.683 \tilde{\Delta}$ and $\left\|\left[O, D^{\prime}\right]\right\|<4 \times 0.683 \tilde{\Delta}$, see (3.6) and the lines that follow. Hence if the point $z q^{m}$ belongs to the segment $\left[D^{\prime}, C^{\prime}\right]$, then this is not the case of the point $z q^{m-2}$, because for $q \in\left(\tilde{q}_{1}, 1 / 2\right]$ one has $q^{-2} \geq 4$ (but one could possibly have $z q^{m-1} \in\left[D^{\prime}, C^{\prime}\right]$ ).

All factors $\left|t_{m}\right|$ of the product $P^{\ddagger} P^{\sharp}$ belong to $\left[b /\left(a^{2}+b^{2}\right)^{1 / 2}, 1\right)$, therefore by Lemma 8, $P^{\ddagger} P^{\sharp} \geq b^{2} /\left(a^{2}+b^{2}\right)$ which for $a \leq b$ is $\geq 1 / 2$.

On the other hand, the moduli of the first three factors $\left|t_{m}\right|$ in $\tilde{P}$ are not less than respectively

$$
132 \tilde{q}_{1}-1>39.814,132{\tilde{q_{1}}}^{2}-1>11.619 \text { and } 132{\tilde{q_{1}}}^{3}-1>2.902
$$

and the moduli of all other factors $\left|t_{m}\right|$ in $\tilde{P}$ (if any) are $\geq 1$, so for $b \geq 132$

$$
\begin{aligned}
\left|\Theta^{*}\right| & \geq e^{-\pi^{2} / 6} \times(39.814 \times 11.619 \times 2.902) \times(1 / 2) \times e^{-2.298978} \times e^{-2 \times 133 / 132^{2}} \\
& >12.8>|G| .
\end{aligned}
$$

Lemma 9. For $a \geq b \geq 132$ one has $\left|\Theta^{*}\right|>|G|$.

Proof. Suppose first that $q \in(1 / 2,1)$. We define $n \geq 3$ from conditions (3.3). Recall that the number $\mu_{1}$ was defined in Lemma 6 and that inequalities (3.8) hold true. For $n \geq 3$ equality (3.11) implies

$$
\begin{aligned}
\mu_{1} & \leq\left(\ln \lambda_{1}\right) 2(n-1)^{2} /(2 n-3)+1 \\
& =\left(\ln \lambda_{1}\right)(n-1 / 2+1 / 2(2 n-3))+1<\left(\ln \lambda_{1}\right) n+0.782,
\end{aligned}
$$

because $1 / 2(2 n-3) \leq 1 / 6$ and $\left(\ln \lambda_{1}\right)(-1 / 2+1 / 6)+1=0.7811 \ldots$

Consider a factor $t_{m}$ from $P_{0}:=\prod_{m=1}^{n} t_{m}$. One has

$$
\left|t_{m}\right|^{2}=\left(a q^{m}-1\right)^{2}+b^{2} q^{2 m} \geq 0.9\left(a^{2}+b^{2}\right) q^{2 m}
$$

this follows from

$$
\left(a^{2}+b^{2}\right) q^{2 m} / 10-2 a q^{m}+1=\left(a q^{m}-10\right)^{2} / 10+\left(b^{2} q^{2 m}-90\right) / 10 \geq 0 ;
$$

the last inequality results from $b \geq 132$ and (3.10) (remember that if $q$ satisfies conditions (3.3) with $n \geq 3$, then the inequality (3.5) holds true), so $b^{2} q^{2 m} \geq$ $(132 / 8)^{2}>90$. Set $A:=\left(a^{2}+b^{2}\right)^{\left(n-\mu_{1}\right) / 2}$. Hence

$$
\begin{aligned}
& \left|P_{0}\right| \geq\left(a^{2}+b^{2}\right)^{n / 2} q^{n(n+1) / 2}(0.9)^{n / 2} \geq\left(a^{2}+b^{2}\right)^{n / 2} 2^{-(n+3)}(0.9)^{n / 2} \\
& P^{\sharp} \quad \geq \quad b^{\mu_{1}} /\left(a^{2}+b^{2}\right)^{\mu_{1} / 2} \\
& \text { so }\left|P_{0}\right| P^{\sharp} \geq A b^{\left(\ln \lambda_{1}\right)(n-2)+1} 2^{-(n+3)}(0.9)^{n / 2}
\end{aligned}
$$


(we use inequalities (3.8)). As $\left(a^{2}+b^{2}\right)^{1 / 2} \geq 132 \sqrt{2}$ and as $n-\mu_{1}>\omega_{1} n-0.782$, $\omega_{1}:=1-\left(\ln \lambda_{1}\right)$, see (3.12), one obtains the minoration $\left|P_{0}\right| P^{\sharp} \geq e^{M}$, where

$$
\begin{aligned}
M:= & \left(\omega_{1} n-0.782\right) \ln (132 \sqrt{2})+(\ln b)\left(\left(\ln \lambda_{1}\right)(n-2)+1\right) \\
& -(n+3) \ln 2+(n / 2) \ln 0.9 .
\end{aligned}
$$

To estimate $P^{\dagger}$ and $P^{\ddagger}$ we use Lemma 5 . As in the proof of Lemma 7 one can minorize the right-hand side of (3.9) by

$$
e^{\left(\pi^{2} / 6\right)(1-n)} e^{M} e^{2(-1.149489 n)} e^{-(b+1) n / b^{2}} .
$$

This expression is of the form $e^{L_{1} n+L_{0}}$ with

$$
\begin{aligned}
L_{1}= & -\pi^{2} / 6+\omega_{1} \ln (132 \sqrt{2})+(\ln b)\left(\ln \lambda_{1}\right) \\
& -\ln 2+(\ln 0.9) / 2-2.298978-(b+1) / b^{2}, \\
L_{0}= & \pi^{2} / 6-0.782 \ln (132 \sqrt{2})+(\ln b)\left(-2 \ln \lambda_{1}+1\right)-3 \ln 2 .
\end{aligned}
$$

For $a \geq b=132$ one has $|z| \geq 132 \sqrt{2}$, also $L_{1}>0.3044>0$ and $L_{0}=-6.0491 \ldots$. For $a \geq b=132, n \geq 3$ one has

$$
L_{1} n+L_{0} \geq-5.136 \ldots>-5.224 \ldots=-\ln (132 \sqrt{2}-1),
$$

i.e. $\left|\Theta^{*}\right|>1 /(|z|-1) \geq|G|$. The functions $L_{1} n+L_{0}$ and $e^{L_{1} n+L_{0}}$ when considered as functions in $b$ (for $n \geq 3$ fixed) are increasing while the functions $-\ln (b-1)$ and $1 /(b-1)$ are decreasing. Therefore one has $\left|\Theta^{*}\right|>1 /(|z|-1) \geq|G|$ for $a \geq b \geq 132$, $n \geq 3$ from which for $q \in(1 / 2,1)$ the lemma follows.

Suppose that $q \in\left(\tilde{q}_{1}, 1 / 2\right]$. One deduces from Lemma 8 (as in the proof of Lemma 7) that $P^{\ddagger} P^{\sharp} \geq b^{2} /\left(a^{2}+b^{2}\right)$. On the other hand, consider the factors $t_{1}$ and $t_{2}$. One can apply to them inequality (3.13) with $m=1$ and 2 . Hence $\left|t_{1}\right|>1$, $\left|t_{2}\right|>1$,

$$
\tilde{P} \geq\left|t_{1}\right|\left|t_{2}\right|>0.9\left(a^{2}+b^{2}\right) \tilde{q}_{1}^{3} \text { and } \tilde{P} P^{\ddagger} P^{\sharp}>0.9 b^{2} \tilde{q}_{1}^{3}>463 .
$$

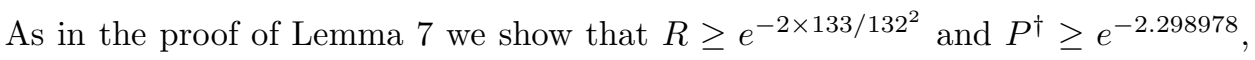
so finally

$$
\left|\Theta^{*}\right|=\tilde{P} P^{\ddagger} P^{\sharp} Q P^{\dagger} R>463 e^{-\pi^{2} / 6} e^{-2.298978} e^{-2 \times 133 / 132^{2}}>8.8>|G| .
$$

Lemmas 7 and 9 together imply that for $q \in(0,1)$ and $z=-a+b i, b>132$, $a>0$, the function $\theta(q,$.$) has no zeros. Theorem 1$ is proved.

\section{REFERENCES}

[1] G. E. Andrews, B. C. Berndt, Ramanujan's lost notebook. Part II. Springer, NY, 2009.

[2] B. C. Berndt, B. Kim, Asymptotic expansions of certain partial theta functions. Proc. Amer. Math. Soc. 139:11 (2011), 3779-3788.

[3] K. Bringmann, A. Folsom, R. C. Rhoades, Partial theta functions and mock modular forms as q-hypergeometric series, Ramanujan J. 29:1-3 (2012), 295-310. http://arxiv.org/abs/1109.6560 
[4] G. H. Hardy, On the zeros of a class of integral functions, Messenger of Mathematics, 34 (1904), 97-101.

[5] J. I. Hutchinson, On a remarkable class of entire functions, Trans. Amer. Math. Soc. 25 (1923), 325-332.

[6] O.M. Katkova, T. Lobova and A.M. Vishnyakova, On power series having sections with only real zeros. Comput. Methods Funct. Theory 3:2 (2003), 425-441.

[7] V. P. Kostov, On the zeros of a partial theta function, Bull. Sci. Math. 137, No. 8 (2013) 1018-1030.

[8] V. P. Kostov, On the double zeros of a partial theta function, Bull. Sci. Math. 140, No. 4 (2016) 98-111.

[9] V. P. Kostov, On the multiple zeros of a partial theta function, Funct. Anal. Appl. 50, No. 2 (2016) 153-156).

[10] V. P. Kostov and B. Z. Shapiro, Hardy-Petrovitch-Hutchinson's problem and partial theta function, Duke Math. J. 162, No. 5 (2013) 825-861.

[11] I. V. Ostrovskii, On zero distribution of sections and tails of power series, Israel Math. Conf. Proceedings, 15 (2001), 297-310.

[12] M. Petrovitch, Une classe remarquable de séries entières, Atti del IV Congresso Internationale dei Matematici, Rome (Ser. 1), 2 (1908), 36-43.

[13] A. Sokal, The leading root of the partial theta function, Adv. Math. 229:5 (2012), 2603-2621. arXiv:1106.1003

[14] S. O. Warnaar, Partial theta functions. I. Beyond the lost notebook, Proc. London Math. Soc. (3) 87:2 (2003), 363-395.

Université Côte dAzur, CNRS, LJAD, France

E-mail address: vladimir.kostov@unice.fr 